\title{
Novel Type of EV Hybrid Traction Drives
}

\author{
Andrey V. Brazhnikov, Nikolay N. Dovzhenko, Andrey N. Minkin, \\ Oksana V. Pomolotova, Anna I. Litvinenko and Viktoriya A. Shilova \\ Siberian Federal University, P.O. Box 16142, Krasnoyarsk 660001, Russia \\ multypha@mail.ru
}

\begin{abstract}
The increase of the phase number of asynchronous electric drive system of vehicles allows not only to improve a number of its technical-and-economic characteristics, but also to create the hybrid traction drives according to scheme "AC Diesel-generator - Commutator - AC induction motor(s)" which will differ fundamentally from the existing ones, and have more simple design and less energy losses (in comparison with analogous existing hybrid drives). These AC hybrid traction drives of novel type are based on the use of the multiphase Diesel generators and non-traditional controlled multiphase induction motors.
\end{abstract}

Keywords: Hybrid traction system; multiphase electrical machine; novel design versions; non-traditional motor control

\section{Introduction}

At present the hybrid traction drives based on the 3-phase induction motors have found a wide application in the field of vehicles of different types (i.e. ground, marine, air vehicles). Among the grave disadvantages of them is a high energy loss in inverter which energizes the 3-phase induction motor (or motors). The existence of these losses is explained by high switching frequency of inverter transistors.

To eliminate this disadvantage it is necessary to use the radically new principles of hybrid AC traction systems designing. According to these novel principles the above mentioned systems must not contain any power electronic elements operating with high frequency.

The creation of such novel hybrid traction systems is possible on the base of multiphase (i.e. having the number $m$ of phases more than four) AC generators (for example, Diesel generators) and non-traditional controlled multiphase induction motors [1].

This paper is a revised and expanded version of a paper No 1569848593 entitled "Peculiarities of Novel Generation of Hybrid Traction Systems for Electrical Vehicles" presented at Workshop on Electrical Engineering 2013 Second (WElectrical2'2013), December 11-13, 2013 at Jeju National University International Center, Jeju Island, Korea.

\section{Main Design Principles of Novel Generation of Hybrid Traction AC Drives}

The prospects for the use of multiphase (i.e. having the number $m$ of phases more than four) asynchronous linear and non-linear drives in the field of vehicles of different types (i.e. ground, marine, air) were presented and described by the authors of this work in a number of their papers (for example, [2]).

However the increase of the phase number of asynchronous electric drive system allows not only to improve a number of its technical-and-economic characteristics, but also to create 
the hybrid traction drives according to scheme "Diesel-generator - AC induction motor(s)" which will differ fundamentally from the existing ones, and have more simple design and control unit and less energy losses (in comparison with analogous existing hybrid drives). These hybrid traction drives of novel type are based on the use of the multiphase Diesel generators and non-traditional controlled multiphase AC induction motors [3].

The basic principles of design of these hybrid traction drives of novel generation are presented in this paper. These basic design principles are the following:

1) the increase of Diesel generator and $\mathrm{AC}$ induction motor phase numbers more than four;

2) the application of such non-traditional methods of control by multiphase AC induction motor as the over-synchronous control method (OSM) and the phase-pole one (PPM) which are the kinds of the over-phase control method (OPM) [4];

3 ) the use of a number of novel design versions of multiphase $\mathrm{AC}$ induction motor.

PPM-controlled multiphase AC induction motor is in effect a multi-speed motor having only one winding set that is equal (in its complexity) to the winding set of 3-phase singlespeed AC induction motor. The quantity of discrete values of the motor speed rises if the phase number of PPM-controlled multiphase AC induction motor increases.

Because of this there is no need to use a transistor inverter in such multiphase hybrid traction drive to regulate the AC induction motor rotor speed. The corresponding thyristor or electromechanical commutator is used in the multiphase hybrid traction drive of novel type instead transistor inverter. The operating frequency of such commutator is much less than the operating frequency of above mentioned inverter IGBTs. Owing to this the energy losses are much less and the motor control process is significantly simpler in the multiphase hybrid traction drive of novel generation (in comparison with the analogous existing hybrid drives) [1].

The combined application of OSM and PPM are necessary for obtaining of the following:

1) wide range of the motor speed regulation;

2) small spacing (step) of the stepwise regulation of the motor speed.

\section{Structure of the Proposed Traction Drive System}

The functional diagram of the power section of the proposed hybrid traction drive system is presented in Figure 1.

In this hybrid drive system the change of motor speed is reached by the change in the version of connection output terminals of m-phase Diesel generator $D G$ to the terminals of the stator phase windings of a $\mathrm{m}$-phase induction motor $M$. This change is reached by the phase commutator $P C$ according to the corresponding signals which are derived from the control unit $C U$ of the proposed hybrid traction drive system.

The mode of operation of the multiphase Diesel generator is optimal constantly.

\section{Peculiarities of the Over-Phase Control Method by Multiphase AC Induction Motors}

\subsection{General Description of the Over-Phase Control Method by Multiphase AC Induction Motors}

The essence of the control according to OPM is that in this case the electrical angles $\alpha$ between the voltages (or currents) of the nearest (in time) phases of the generator are 
increased by a factor of $H$ (in comparison with any traditional control method) without any change of the generator voltage (or current) amplitude and frequency, i.e., in this case $\alpha_{H}=H$ - $\alpha_{T}$, where $H$ is some whole number, $\alpha_{T}$ is the value $\alpha$ when some traditional control method is used $\left(\alpha_{T}=2 \pi / m\right)$, and $\alpha_{\mathrm{H}}$ is the value $\alpha$ when OPM is used (see Figures 2 and 3).

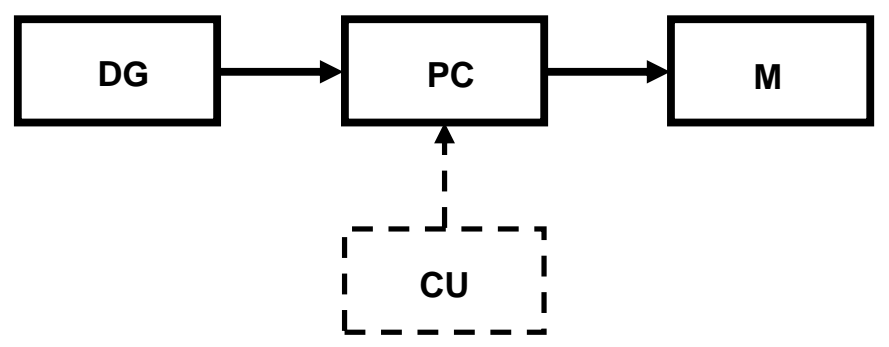

Figure 1. Functional diagram of the power section of the proposed hybrid traction drive system, where $D G$ is $m$-phase Diesel generator, $P C$ is thyristor or electromechanical phase commutator, $M$ is $m$-phase induction motor, and $C U$ is control unit of the hybrid traction drive system

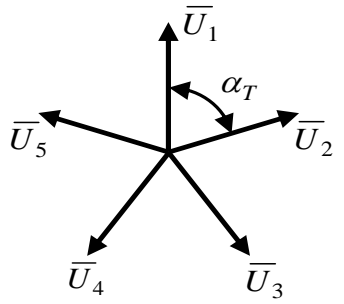

a)

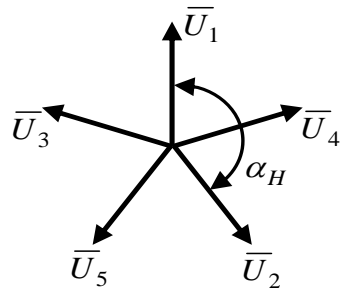

b)

Figure 2. 5-phase system of the generator output voltages: (a) $H=1$, (b) $H=2$

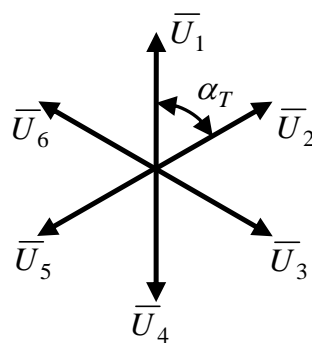

a)

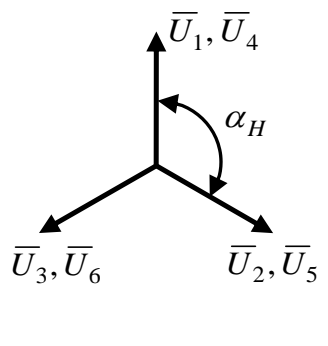

b)

Figure 3. 6-phase system of the generator output voltages: (a) $H=1$, (b) $H=2$

The change of this phase shift can be obtained by the use of the above mentioned electronic or mechanical phase commutator is placed between a multiphase Diesel generator and multiphase induction motor. In this case the change in the OPM parameter $H$ is reached by the change in the version of connection output terminals of the generator to the terminals of the motor stator phase windings. 
The coefficient $H$ is the major parameter of OPM that characterizes the type of this control method (the value $H=1$ corresponds to any traditional control method, and the value $H>1$ corresponds to OPM).

The range of the parameter $H$ (including its maximal value), which can be achieved in the given drive system, depends on the phase number of the system and on a motor stator winding type.

The change of the parameter $H$ results in the change of the filtering properties of the generator multiphase induction motor drive. In particular, the numbers of the harmonics, which take part in the creation of the magnetic field in a motor air gap, are described by the following equation:

$$
H \cdot c \pm n / p=b \cdot m
$$

where $c$ is the number of the phase voltage (or current) harmonic (i.e. the number of the time harmonic), $n$ are the numbers of the harmonics of the functions which describe a space distribution of the mutual inductances between motor phase windings (i.e. the numbers of the space harmonic), the coefficient $b=0, \pm(1,2,3, \ldots)$, and $p$ is the number of motor poles pairs.

The magnetic fields created in a motor air gap by the harmonics, numbers of which do not satisfy (1), cancel each other.

Formula (1) is derived as a result of solving the equations that describe the electromagnetic processes taking place in $\mathrm{m}$-phase induction motor [5].

OPM has two kinds - OSM and PPM. The use of these OPM kinds allows achieving the following two effects:

- the increase of the motor movable secondary element motion speed over its synchronous value $V_{S}$ which is conditioned by the generator output voltage frequency and the motor pole number (OSM),

- the increase of the motor linear forces (or torques) without any change in the amplitude and frequency of the generator output voltage (PPM) [2, 4].

OSM differs from PPM in the following: when OSM is used, the spectrum of the function, which describes the space distribution of the mutual inductances between motor phase windings, does not change for all parameter $H$ values which have to be realized in the given drive system. When PPM is used, the spectrum of the above-mentioned function changes during $H$ value changing process.

The peculiarities of OSM and PPM are described in [2, 4].

\subsection{Peculiarities of OSM}

When OSM is used, the motor movable secondary element motion speed $V$ may be increased by a factor of some whole number $C_{O}$ over its synchronous value $V_{S}$, i.e. $V=C_{O}$. $V_{S}$ (without any change in the motor pole length and generator output voltages frequency), where $C_{O}=(m \pm 1) / H$.

As an example mechanical characteristics of a multiphase induction motor are shown in Figure 4 on a per-unit basis for the cases when the traditional control method (line 1) and OSM (line 2) are used for the case when $P_{1}=$ const at $H=$ var, where $F$ is a linear traction force (or torque, when the motor stator is bow-shaped or the motor is of rotation type), $P_{1}$ is the drive system power input. 


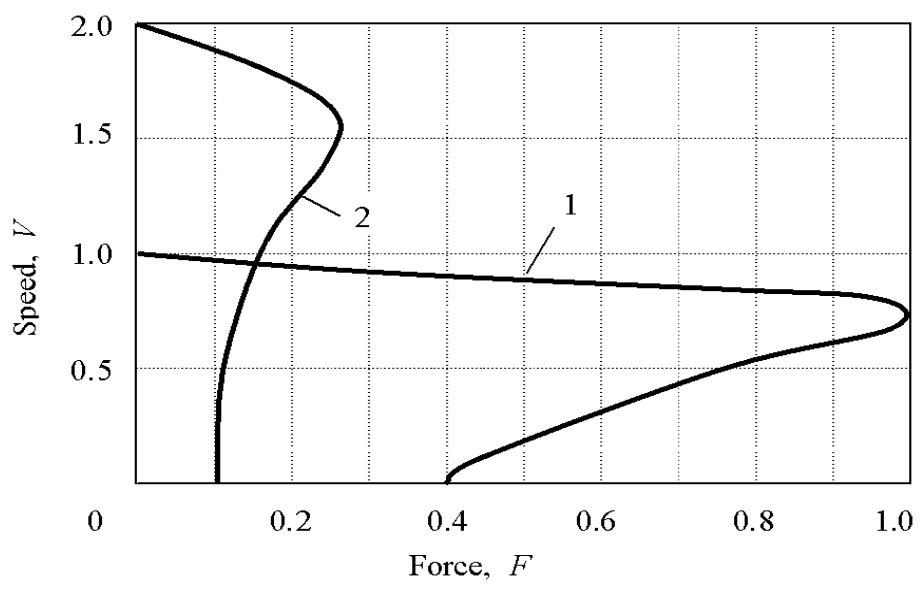

\section{Figure 4. Mechanical characteristics of a multiphase generator-fed induction motor on a per-unit basis: (1) natural characteristic, when the traditional control method is used $(H=1)$, and (2) when OSM is used $(H=2)$}

The increase of the motion speed $V$ over the value $V_{S}$ during the use of OSM is explained by the following. When OSM is used, the distribution of the energy of the above mentioned time harmonics over space ones, in the process of energy interchange between a motor stator and rotor winding sets, changes in comparison with the case when $H=1$, i.e., the degree of time harmonics contribution to the creation of the total magnetic field in a multiphase induction motor air gap changes. In this case one or other higher harmonic (but not the first one) becomes the major harmonic that makes the greatest contribution to the creation of the magnetic field and sets the speed of its motion along the length of the air gap.

For example, if $m=5$ and $H=2$, the magnetic induction in the motor air gap is largely created by the third harmonic ( $c==3$ ) of the motor phase voltages (currents). The energy of the first time harmonic is largely expended for losses on the multiphase motor stator winding set.

It has been known that in real situations (i.e., in real generator phase voltage spectra) the higher time harmonics have less energy than the first one. These phenomena explains both the increase of the motion speed $V$ over the value $V_{S}$, and the decrease of the motor efficiency if OSM is used.

When OSM is used, the number of poles of the magnetic field in the motor air gap does not change and has the same value that it has at $H=1$.

As an illustration, Figures 5 and 6 show the space-temporal diagrams of the magnetic induction in a motor air gap, which is created by the stator winding set of the 5-phase induction motor in the case when:

- the type of the stator phase currents is right-angled meander,

- the number of the motor poles pairs $p=1$,

- the number of the motor stator slots $Z=10$,

- the number of the stator slots per phase and pole $q=2$,

where $t$ is time, space coordinate axis $O \gamma$ runs along entire length of the linear or nonlinear motor air gap $(0 \leq \gamma \leq 2 \pi), B$ is the magnetic induction created by the motor stator winding set in the motor air gap, $B_{m}$ is maximal value of the magnetic induction $B, B_{O}$ is the magnetic induction created by one phase winding of the motor stator in the motor air gap. 


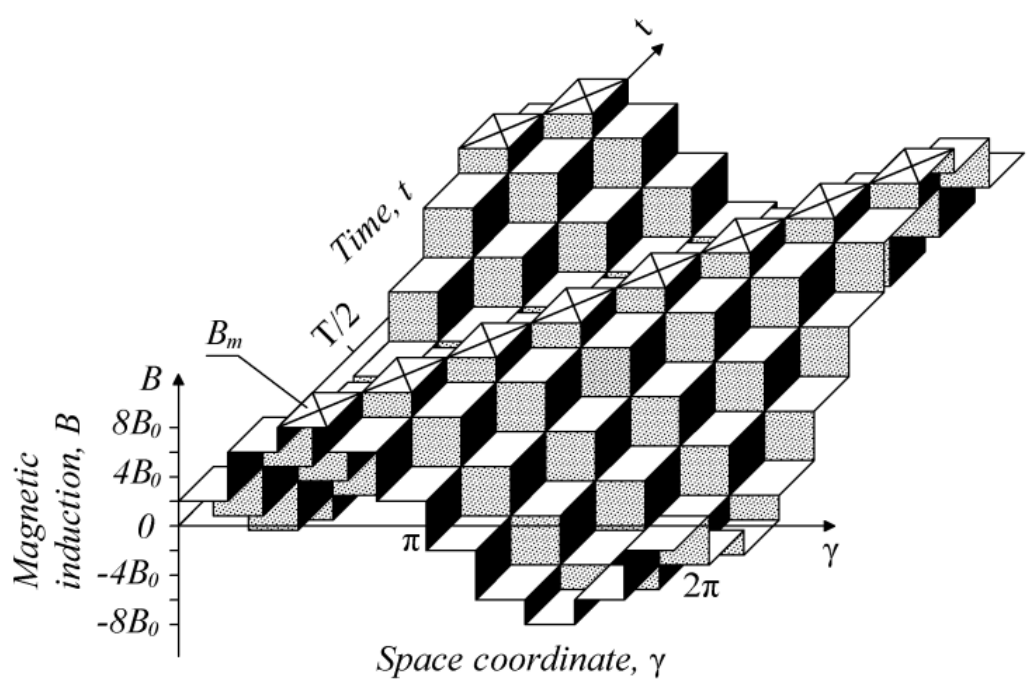

Figure 5. Space-temporal diagram of the magnetic induction in a motor air gap, which is created by the stator winding set of the 5-phase induction motor at $H$ $=1$ (traditional control)

From these diagrams it is obvious that when $m=5$ and $H=2\left(C_{O}=3\right)$ the motion speed of the maximum of the magnetic induction $\mathrm{B}$ is three times more than that at $H=1\left(C_{O}=1\right)$, and the above mentioned maximum has time to pass three times along entire length of the motor air gap during one period of the generator output voltage. The number of the poles of the magnetic field created by the motor stator winding set in the motor air gap does not change in the case when OSM is used.

\subsection{Peculiarities of PPM}

During PPM application process, when the parameter $H$ changes, the effect adequate to the synchronous change of the drive phase number and number of motor poles appears.

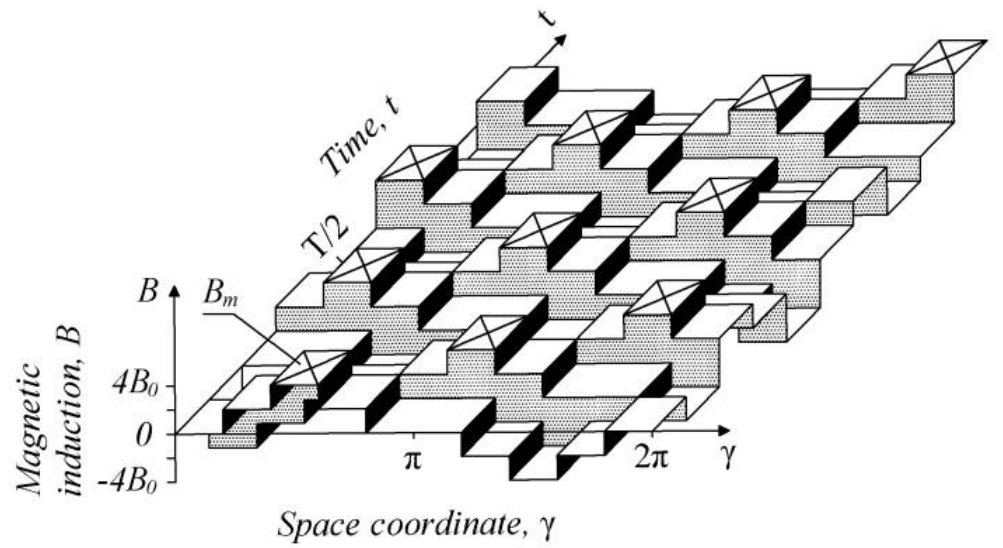

Figure 6. Space-temporal diagram of the magnetic induction in a motor air gap, which is created by the stator winding set of the 5-phase induction motor at $H$ $=2$ (OSM) 
PPM being used, the following equation is fulfilled:

$$
p_{e} \cdot m_{e}=p \cdot m=\text { const, }
$$

where $m$ is real value of the phase number of the multiphase induction motor drive system, $m_{e}$ is equivalent (observed) value of the drive system phase number, $p$ is real number of the motor poles pairs, and $p_{e}$ is equivalent (observed) number of the motor poles pairs $\left(p_{e}=p \cdot H\right)$.

Because of this when PPM is used, the maximal torque (linear force) of an induction motor is directly proportional to the parameter $H$ value, and the idle speed of the motor movable secondary element (rotor) is inversely proportional to the parameter $H$ (without any change in the motor pole length and generator output voltages frequency), i.e. $V=V_{1} / H$, where $V_{1}$ is the motor movable secondary element motion speed $V$ if $H=1$.

Thus the use of PPM allows to change the motor speed $V$ without any change in the generator output voltages frequency.

\section{Design Peculiarities of the Power Units of the Proposed Hybrid Traction Drive System}

\subsection{Design of Diesel Generator}

The number of phases of the AC Diesel generator is more than four $(m>4)$. It is its sole constructive difference from traditional 3-phase AC Diesel generator. The winding set of the $\mathrm{m}$-phase Diesel generator is of traditional drum type. The number $\mathrm{m}$ of phases of this winding set must be equal to the phase number of the AC induction motor.

\subsection{Commutator}

Phase shifts between of the nearest phase voltages of the motor must be increased by a factor of some whole number when OPM is used. Because of this the transition from traditional control to OPM is possible by the change in order of the connection between the motor phase windings and motor power supply phases (for example, generator phases) by application of the commutator (without any use of an inverter).

The electronic or mechanical phase commutator $P C$ (see Figure 1) must connect output terminals of generator to the terminals of the motor stator phase windings according to the following algorithm:

$$
i_{M}=N_{1}-N_{2}
$$

where $N_{1}=H \cdot\left(i_{i n}-1\right)+1, N_{2}=0$ if $H=1, N_{2}=m \cdot\left[\left(N_{1}-1\right) / m\right]$ if $H \geq 2, i_{M}$ is the number of a motor stator phase winding, $i_{\text {in }}$ is the number of an Diesel generator phase, and $\left[\left(N_{1}-1\right) / m\right]$ is the integer part of the number $\left(N_{1}-1\right) / m$.

The peculiarities of the phase commutator $P C$ may be conveniently shown by the example of the following case (see Figure 7): 


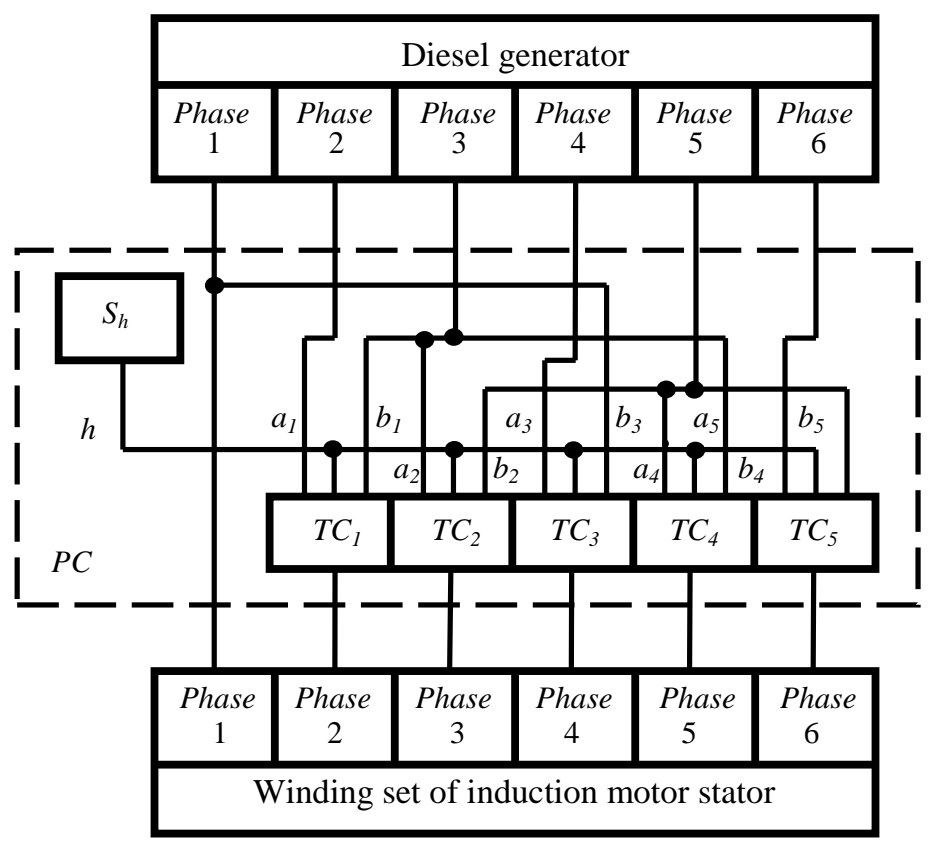

Figure 7. Structural diagram of the 6-phase single-motor OPM-controlled system "Diesel generator - induction motor" with phase commutator between Diesel generator and induction motor, where $S_{h}$ is the source of the signal $h$, and $T C$ are the electronic or mechanical terminal changers

$-m=6$,

- 6-phase inverter has traditional scheme and operates at the sole value of the parameter $H$ : $H=1$,

- the change in the parameter $H$ value is achieved by phase commutator $P C$,

- the parameter $H$ may be equal to 1 or 2 ,

- the logic signal $h$ depends on the value of the OPM parameter $H$ (i.e. $h=1$ if $H=1$, and $h=0$ if $H=2$ ),

- and $T C_{j}$ is the electronic or mechanical terminal changer for the phase having number $j=i+1, i \in[1 ; m], j \in[1 ; m-1]$.

If the drive system is low-powered, the type of electronic terminal changers $T C$ may be logic. The electronic logic version of the terminal changer $T C_{j}$ is shown in Figure 8 (for the case of low-power drive system), where the unit "\&" is an AND-type logic gate, and the unit " $\geq 1$ " is an OR-type logic gate. The mechanical switching analogue of the logic scheme shown in Figure 8 is presented in Figure 9. 


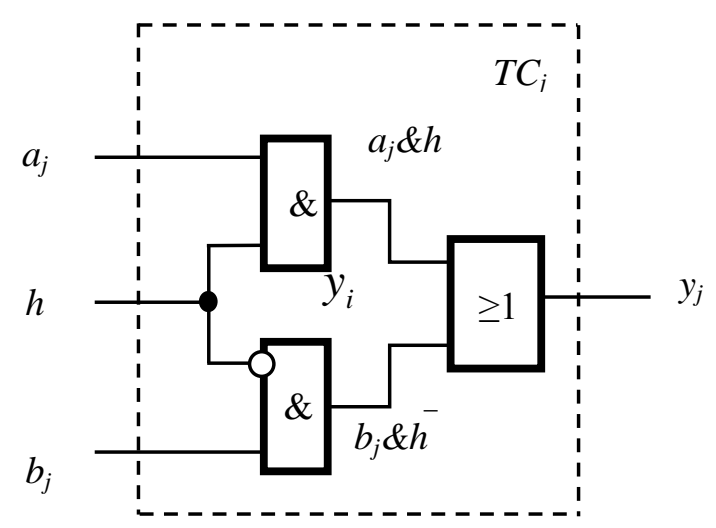

Figure 8. Logic version of the electronic terminal changer $T C_{j}$

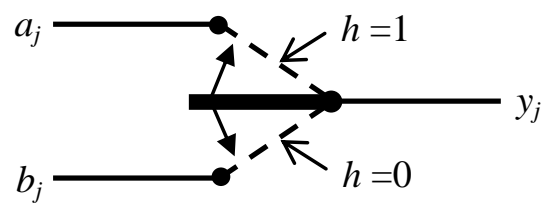

\section{Figure 9. Mechanical switching analogue of the logic scheme shown in} Figure 8

These schemes carry out the following logic function:

$$
y_{j}=a_{j} \& h \vee b_{j} \& \bar{h}=\left\{\begin{array}{lll}
a_{j} & \text { if } h=1 ; \\
b_{j} & \text { if } h=0 .
\end{array}\right.
$$

\subsection{Design peculiarities of the Multiphase PPM-Controlled Induction Motor}

The analysis of the equations of the multiphase drives mathematical models [5] shows that the following necessary conditions must be fulfilled for attaining the opportunity of PPM realization without any deterioration of drive system technical-and-economic characteristics (in particular, system efficiency) in comparison with the case when $H=1$ :

Condition 1. The harmonics having numbers $n_{m .1}=p \cdot H$ must be contained in the spectra of the functions describing the space distribution of the mutual inductances between AC motor phase windings for all parameter $H$ values which have to be realized in the given drive system.

Condition 2. When $H>1$, the amplitudes of the above-mentioned harmonics (i.e., having numbers $n=n_{m .1}$ ) must have the values being not less than those at $H=1$.

It is obviously that the fulfillment of these conditions (i.e. the above mentioned law of $\mathrm{H}$ invariance) may be provided only with obtaining the conductive decoupling between the AC motor stator slots, which cannot be achieved if design of the motor and its stator winding set are traditional.

Because of this it is necessary to use some peculiar designs of multiphase AC liner and non-linear motors to ensure fulfillment of the $H$-invariance law [6, 7]. 
According to this deduction the following a number of three basic design versions were developed by the authors of this paper:

Basic design version 1 (BDV-1). The AC motor stator has toroidal-type winding set. The motor rotor envelops the stator both on the outside and on the inside (when the motor is of rotating type) or from above and from below (when the motor is linear or bow-shaped).

Basic design version 2 (BDV-2). The AC motor stator winding set consists of the individual rods located in stator slots and insulated from stator magnetic circuit.

Basic design version 3 (BDV-3). The AC motor stator core (i.e. magnetic circuit) consists of two parts magnetically separated from each other by diamagnetic shield.

The application of any one of these design versions allows to achieve the fulfillment of the $H$-invariance law $[6,7]$.

The design of multiphase induction motor of rotating type worked out according to the basic design version BDV-1 is shown in section in Figure 10. This motor has toroidal-type stator winding set and $E$-shaped rotor enveloping the motor stator both on the outside and on the inside. The motor stator has two rows of slots: the first row is located on the inner surface of the stator core (the inner stator slots), and the second row is located on the outer surface of it (the outer stator slots). The slots disposition on the stator surface of the 8-phase induction motor developed according to the basic design version BDV-1 is shown in Figure 11. Every stator phase winding drops in radial direction in two slots - in one inner slot and in one outer slot $[5,6]$.

Exterior view of the 24-phase induction motor stator, which is developing according to the drawing shown in Figure 16, is shown in Figure 12.

The various versions of the $E$-shaped rotor design of such induction motor can be used. For example, this rotor may have solid ferromagnetic body with $U$-shaped axial-and-radial slots made on the rotor surface according for the decrease of the electrical losses in the rotor circuit (see Figure 13) $[8,9]$.

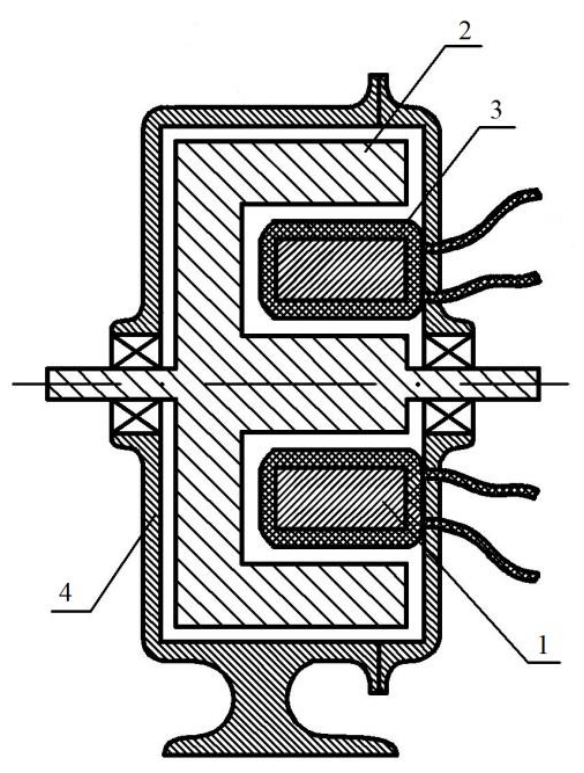

Figure 10. The section of multiphase induction motor of rotating type worked out according to the basic design version BDV-2, where 1 is the stator core 
(i.e., magnetic circuit), 2 is the rotor core (magnetic circuit), 3 is a stator phase winding, and 4 is the motor case

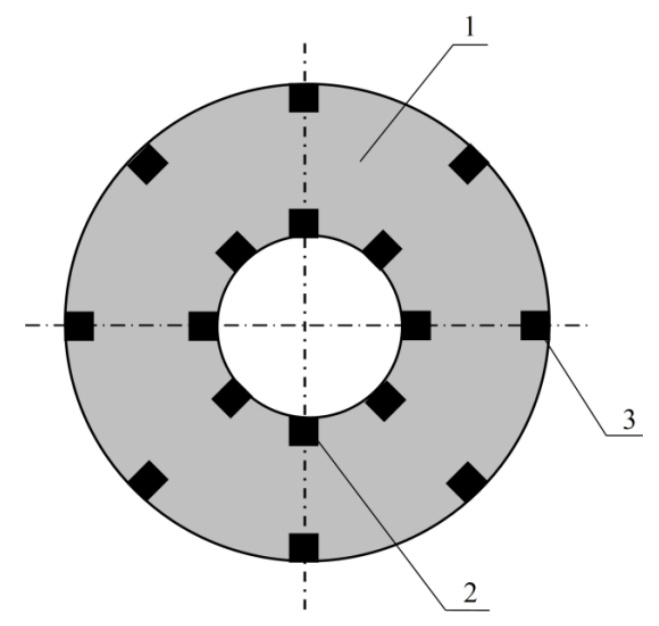

Figure 11. The slots disposition on the stator surface of the 8-phase induction motor developed according to the basic design version BDV-2, where 1 is the stator core (i.e. stator magnetic circuit), 2 is the an inner stator slot, and 3 is an outer stator slot

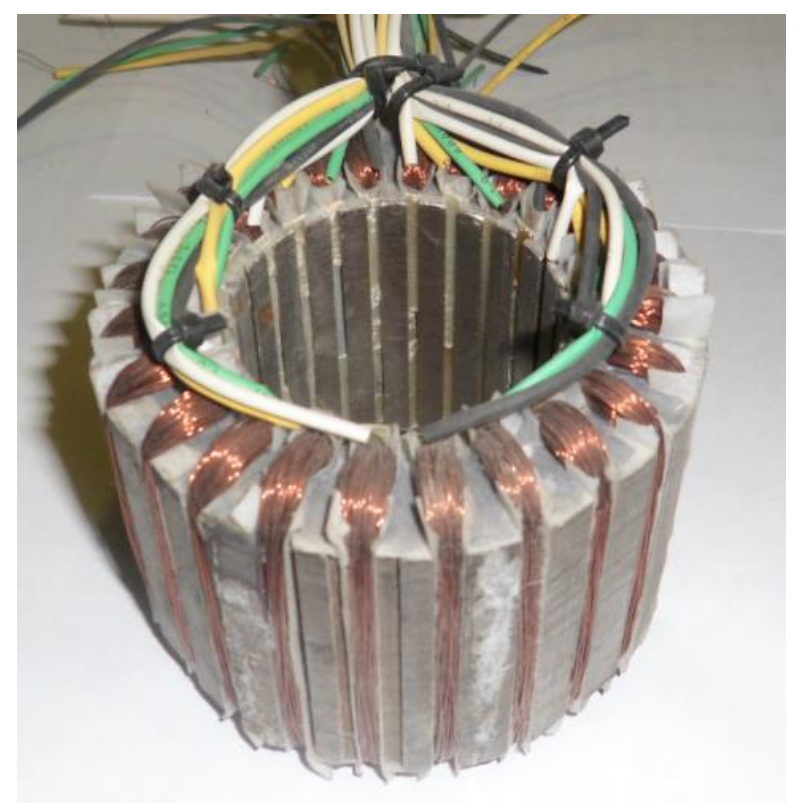

Figure 12. Exterior view of the 24-phase induction motor stator developing according to the drawing shown in Figure 11 


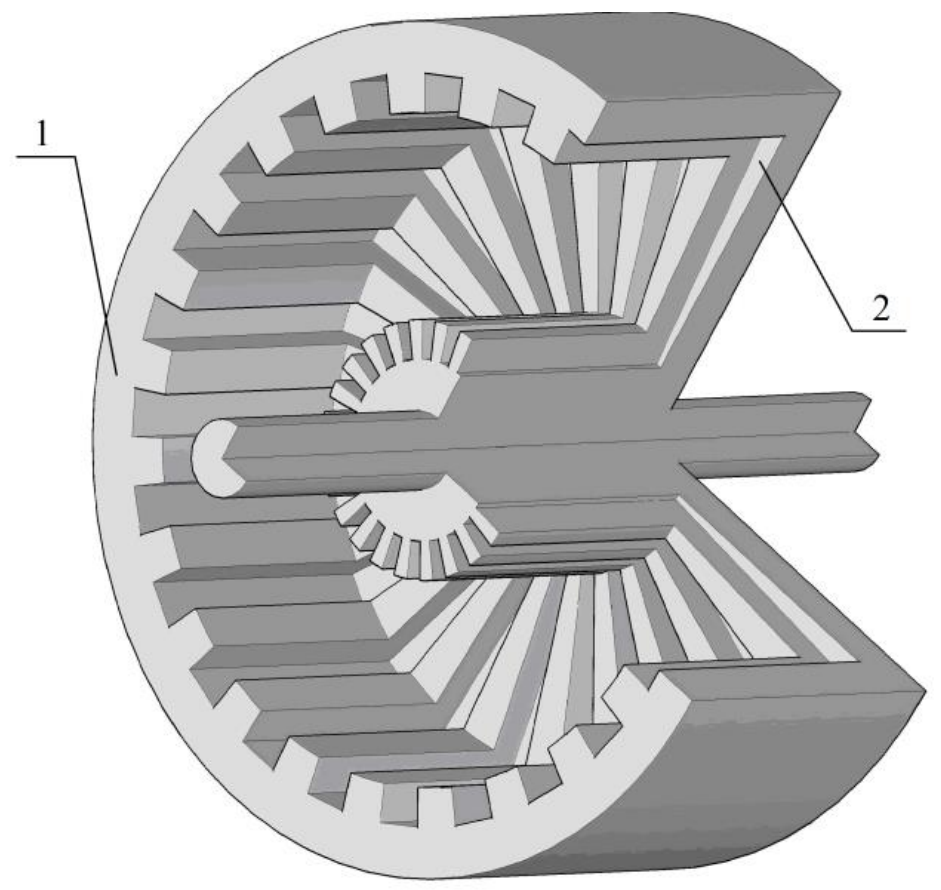

Figure 13. E-shaped solid ferromagnetic rotor with $U$-shaped axial-and-radial slots, where 1 is the body of the rotor, and 2 is a $U$-shaped axial-and-radial rotor slot

\section{Possibility to Decrease the Step of Speed Changing}

The main disadvantage of the proposed hybrid drive system is the stepwise change of the motor speed.

However it is possible to obtain some quasi-continuous change of this speed by the decrease in the step of its change. This speed change step decrease can be obtained by the use of the following methods:

Method 1. By the choice of some great value of the parameter $H$ (not $H=1$, but $H>>1$ ) as the nominal value of this parameter.

Method 2. By the use of not 1-, but 2-stage (2-level) commutator [10] shown in Figure 14. In this case the 1st commutator stage implements the transition to PPM, and the 2nd commutator stage implements the transition to OSM. The combined operation of both commutator stages will allow to decrease the motor speed change step. 


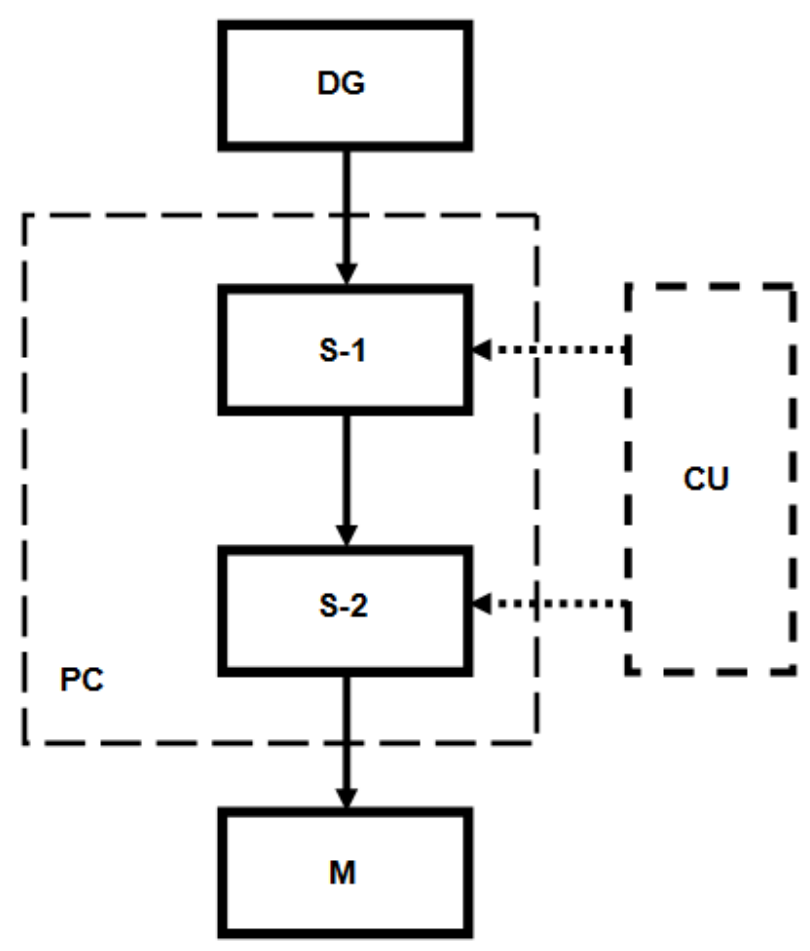

Figure 14. Functional diagram of the power section of the proposed hybrid traction drive system having 2-stage commutator, where $S-1$ is the 1st stage of $P C$, and $S-2$ is the 2nd stage of $P C$

Method 3. By the combined use of the above mentioned two methods (the method 1 and method 2).

\section{Conclusion}

The increase of the phase number of asynchronous electric drive system allows not only to improve a number of its technical-and-economic characteristics, but also to create the hybrid traction drives according to scheme "Diesel-generator - AC induction motor(s)" without application of any power electronic elements operating with high frequency, which will differ fundamentally from the existing ones, and have more simple design and control unit and less energy losses (in comparison with analogous existing hybrid drives).

\section{References}

[1] A. Brazhnikov, N. Dovzhenko, A. Minkin, O. Pomolotova, A. Litvinenko and V. Shilova, "Peculiarities of Novel Generation of Hybrid Traction Systems for Electrical Vehicles”, Adv. Sci. Technol. Lett., vol. 37 (Electr. Eng. 2013), Jeju Island, Korea (2013), pp. 17-20.

[2] A. V. Brazhnikov and I. R. Belozerov, "Prospects for the Use of Multiphase Inverter-Fed Asynchronous Drives in the Field of Traction Systems of Railway Vehicles”, Int. J. of Railw., vol. 5, no. 1, (2012), pp. 3847.

[3] A. V. Brazhnikov, "Designing Principles of Novel Generation of Hybrid Drives with AC Induction Motors for Railway Transport", Mod. Probl. of Sci. and Educ., No 6 (Suppl. Tech. Sci.), (2012), pp. 21.

[4] A. V. Brazhnikov and I. R. Belozerov, "Over-Phase Control of Inverter Multiphase AC Linear Drives", Mechatron., vol. 23, issue 2, (2013), pp. 227-232.

[5] A. V. Brazhnikov, "Multiphase Inverter Electric Drive with Different Versions of Induction Motor Rotor", Ph.D. Thesis, Dept. Electr. Mach. and Drives, Tomsk Politech. Inst., Tomsk, Russia, (1985) (in Russian). 
[6] A. V. Brazhnikov and E. S. Brazhnikova, "Efficiency Invariance Laws and Development of Multiphase AC Inverter Drives", Proceedings of 21st International Symposium on Power Electronics, Electrical Drives, Automation and Motion SPEEDAM'2012, Sorrento, Italy, (2012), pp. 420-425.

[7] A. V. Brazhnikov, and I. R. Belozerov. Space-Temporal Spectral Relations and Energy Efficiency Invariance Laws Acting in the Field of Inverter-Fed Multiphase AC Drives. Proceedings of IET 6th International Conference on Power Electronics, Machines and Drives PEMD‘2012, Bristol, Great Britain (2012), paper No 0027, 6 p., CD-ROM.

[8] A.V. Brazhnikov, I.R. Belozerov, and S.A. Molokitin. Solid Ferromagnetic Rotor for Phase-Pole-Controlled Induction Motor. Russian Patent RU 109345U1, Oct. 10 (2011) (in Russian).

[9] A.V. Brazhnikov, and I.R. Belozerov. E-Shaped Rotor Having Short-Circuited Winding for Phase-PoleControlled Induction Motor. Russian Patent RU 118805U1, July 27 (2012) (in Russian).

[10] A.V. Brazhnikov, E.S. Brazhnikova, R.V. Bondarenko, and A.A. Urchukov. Speed Stepwise Controlled Multiphase Induction Motor Drive. Russian Patent RU 130165U1, July 10 (2013) (in Russian).

\section{Authors}

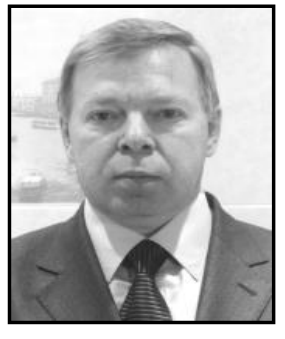

Andrey V. Brazhnikov is presently an associate professor of the Siberian Federal University (Krasnoyarsk, Russia) and a professor of the Russian Academy of Natural History. He had his graduation in Electrical Engineering from the Krasnoyarsk Polytechnical Institute (Krasnoyarsk, Russia) in 1982 (major in Automatics and Telemechanics, Honors Degree, cum laude). He received Ph.D. degree in Electromechanics from the Tomsk Polytechnical Institute (Tomsk, Russia) in 1985. Now he has more than 170 published scientific works and inventions.

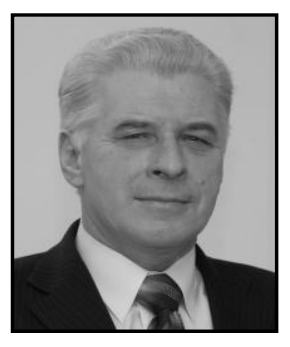

Nikolay N. Dovzhenko, Ph.D., Professor, is presently the Director of the Institute of Petroleum and Natural Gas Engineering and Vice-Rector of the Siberian Federal University (Krasnoyarsk, Russia). He had his graduation in Metallurgy from the Krasnoyarsk Institute of Non-Ferrous Metals (Krasnoyarsk, Russia) in 1973 (major in Processing of Metals by Pressure, Honors Degree, cum laude). He received Grand Ph.D. degree in Metallurgy from the National University of Science and Technology "MISIS" (Moscow, Russia) in 2002. Now he has more than 200 published scientific works and inventions.

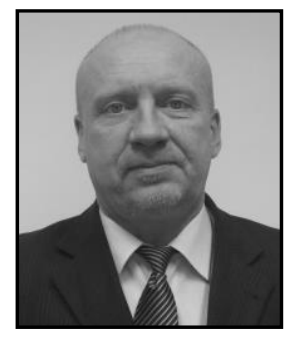

Andrey N. Minkin is presently the Head of the Department of Fire Safety of the Siberian Federal University (Krasnoyarsk, Russia) and a professor of the Russian Academy of Natural History. He had his graduation in Mechanics from the Krasnoyarsk Polytechnical Institute (Krasnoyarsk, Russia) in 1984 (major in Technology of Mechanical Engineering). He received Ph.D. degree in Chemical Mechanical Engineering from the Lomonosov Moscow State University of Fine Chemical Technologies (Moscow, Russia) in 1988. Now he has more than 60 published scientific works and inventions. 


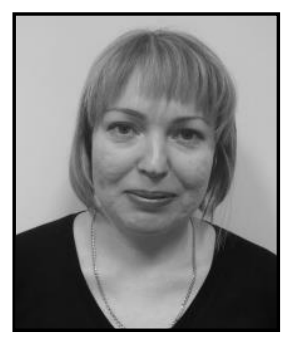

Oksana V. Pomolotova is presently a lecturer and $\mathrm{PhD}$ student of the Siberian Federal University (Krasnoyarsk, Russia). She had her graduation from the Krasnoyarsk State Pedagogical University (Krasnoyarsk, Russia) in 2009.

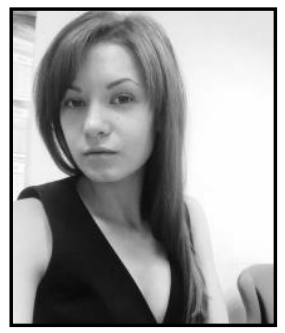

Anna I. Litvinenko is presently a 4th year student and officer of the Educational-and-Organizational Department of the Siberian Federal University (Krasnoyarsk, Russia).

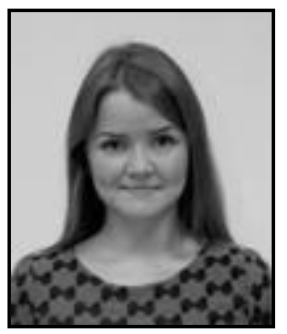

Viktoriya A. Shilova is presently a 4th year student of the Siberian Federal University (Krasnoyarsk, Russia). She is also an officer of the Vankor Oil and Gas Corporation. 
International Journal of Control and Automation Vol.7, No.3 (2014) 